\title{
Der Weg nach Rom - Entstehung und Bedeutung der Römischen Verträge
}

\author{
Wilfried Loth*
}

50 Jahre nach Unterzeichnung der Römischen Verträge am 25. März 1957 besteht vielfacher Anlass, nach dem historischen Ort der Europäischen Union zu fragen. Wie und warum ist sie entstanden? Welche Entwicklungsmuster wurden mit ihrer Gründung angelegt, und was lässt sich daraus für die Bewältigung ihrer aktuellen Probleme ableiten?

Die Geschichtswissenschaft hat sich in den letzten Jahren intensiv und zum Teil auch kontrovers mit diesen Fragen befasst. ${ }^{1}$ Dabei wurde deutlich, dass die gängigen Interpretationsmuster der Geschichte der europäischen Integration zu kurz greifen. Offensichtlich läuft die europäische Integration nicht einfach, wie es eine föderalistisch-universalistische Teleologie angenommen hat, auf den Zusammenschluss der europäischen Nationalstaaten zu einem Bundesstaat des alten Kontinents hinaus. Ebenso wenig, das zeigen die zahlreichen Krisen und Blockierungen, wird sie vorwiegend aus wohlfahrtsstaatlichen Motiven so automatisch zu zunehmender politischer Einheit vorangetrieben, wie die neofunktionalistische Schule behauptet hat. Und angesichts der zunehmenden Eigenständigkeit der europäischen Organe lässt sie sich auch nicht als bloße Fortsetzung nationalstaatlicher Politik mit europäischen Mitteln charakterisieren, wie es eine vermeintlich realistische Betrachtungsweise gerne glauben machen möchte.

\section{Grundlegende Impulse}

Bei einem umfassenden Blick auf die Geschichte des 20. Jahrhunderts erscheint der Prozess der europäischen Integration als eine Antwort auf Funktionsdefizite der Nationalstaaten beziehungsweise des nationalstaatlichen Ordnungssystems in Europa. In den Auseinandersetzungen um die Durchsetzung des nationalstaatlichen Prinzips in der ersten Hälfte des 19. Jahrhunderts war die Schaffung eines europäischen Ordnungsrahmen meist mitgedacht worden; nationale Bewegungen sahen sich im Streben nach Schaffung einer europäischen Gemeinschaft vereint. Erst die Zweckbündnisse dieser Bewegungen mit etablierten Monarchien in der zweiten Jahrhunderthälfte führte zur schrankenlosen Konkurrenz souveräner Nationalstaaten in einem fragilen Gleichgewichtssystem. ${ }^{2}$ Je deutlicher die Defizite dieses Systems dann in der ersten Hälfte des 20. Jahrhunderts hervortraten, desto stärker wurden Bewegungen, die auf eine Relativierung des nationalstaatlichen Ordnungsprinzips zielten. Vier solcher Bewegungen lassen sich unterscheiden. ${ }^{3}$

* Prof. Dr. Wilfried Loth, Historisches Institut, Abteilung für Neuere und Neueste Geschichte, Universität Duisburg-Essen.

1 Vgl. Wilfried Loth: Beiträge der Geschichtswissenschaft zur Deutung der Europäischen Integration, in: Wilfried Loth / Wolfgang Wessels (Hrsg.): Theorien europäischer Integration, Opladen 2001, S. 87-106; Jost Dülffer: Europäische Integration zwischen integrativer und dialektischer Betrachtungsweise, in: Archiv für Sozialgeschichte, Band 42, Bonn 2002, S. 521-544; Jost Dülffer: Europäische Zeitgeschichte - Narrative und historiographische Perspektiven, in: Zeithistorische Forschungen 1/2004, S. 51-71.

2 Vgl. hierzu zuletzt Jürgen Elvert: Die europäische Integration, Darmstadt 2006, S. 12-29.

3 In dieser Systematik erstmals vorgestellt bei Wilfried Loth: Der Prozess der europäischen Integration. Antriebskräfte, Entscheidungen und Perspektiven, in: Jahrbuch für Europäische Geschichte 1/2000, S. 17-30. Als Gesamtdarstellung, die diese Motive entfaltet, vgl. Wilfried Loth: Der Weg nach Europa. Geschichte der europäischen Integration 1939-1957, Göttingen 1995. 
Zunächst das Problem der zwischenstaatlichen Anarchie, Auslöser aller ,klassischen Friedenssicherungspläne von Dante bis Kant: Es wurde immer drängender, seit die Entwicklung der modernen Kriegstechnik Millionen von Menschen zu Kriegsopfern werden ließ und die wirtschaftlichen Schäden im Zeitalter der Kabinettskriege ungeahnte Ausmaße annahmen. Die Erfahrung des Ersten Weltkriegs führte darum zu einer Fülle europäischer Friedensinitiativen, von denen der Paneuropa-Feldzug des Grafen Coudenhove-Kalergi und Briands Europaplan nur die spektakulärsten waren. Als die Friedensordnung von Versailles ab 1938 schrittweise zerbrach, erhielt diese Bewegung einen weiteren Schub. „Man kann es vor aller Welt mit tiefster und unbeugsamster Überzeugung aussprechen," schrieb etwa Léon Blum im Frühjahr 1941: „Aus diesem Krieg müssen endlich durch und durch starke internationale Einrichtungen und eine durch und durch wirksame internationale Macht hervorgehen, sonst wird er nicht der letzte gewesen sein. "4

Ein besonderer Aspekt des Problems der Friedenssicherung war die deutsche Frage: Wie die stärkste Nation in der Mitte des europäischen Kontinents sich entfalten lassen, ohne gleichzeitig unter eine Hegemonie der Deutschen zu geraten? Oder umgekehrt: Wie die Deutschen kontrollieren, ohne durch einseitige Diskriminierung neuen Revanchismus hervorzurufen? „Um den Widerspruch zu lösen“, so wiederum Blum stellvertretend für viele Autoren des Widerstands, ,um die Unschädlichkeit Deutschlands in einem friedlichen und gesicherten Statut zu erreichen, gibt es einen einzigen Weg: die Eingliederung der deutschen Nation in eine internationale Gemeinschaft ${ }^{* 5}$ - also beispielsweise: nicht nur eine Kontrolle des Ruhrgebiets, sondern eine gemeinsame Lenkung der gesamten europäischen Schwerindustrie, nicht nur eine Beschränkung der deutschen Militärhoheit, sondern ein gemeinsames Kommando für alle europäischen Streitkräfte.

Ein drittes Funktionsdefizit des Nationalstaatensystems ergab sich aus der Entwicklung der Produktivkräfte des industriellen Zeitalters: Die nationalen Märkte in Europa wurden, je länger, desto deutlicher, für rationale Produktionsweisen $\mathrm{zu}$ eng. Ihre wechselseitige Abschottung war nur temporär und sektoral sinnvoll, langfristig führte sie zu einem Verlust an Produktivität. Das hatte einen volkswirtschaftlichen und einen machtpolitischen Aspekt; vor allem in Gestalt der US-amerikanischen Konkurrenz waren beide seit den 1920er Jahren präsent. Entsprechend zahlreich waren auch die Einigungsinitiativen im wirtschaftlichen Bereich. Auch hier sorgte die Erfahrung des Zweiten Weltkriegs für einen zusätzlichen Motivationsschub: Während die Europäer ihre Ressourcen in diesem Krieg weitgehend verschlissen, weiteten die USA ihr Produktionsvolumen um mehr als das Doppelte aus, durch ihre Funktion als wichtigster Materiallieferant der Anti-Hitler-Koalition ebenso begünstigt wie durch die Abwesenheit der europäischen Länder vom Weltmarkt.

Ein vierter Motivationskomplex für europäische Einigungsinitiativen ist damit ebenfalls schon angesprochen: Das Streben der Europäer nach Selbstbehauptung gegenüber den neuen Weltmächten. Sowohl die Sorge vor einer wirtschaftlichen und politischen Übermacht der USA als auch die Furcht vor einer Expansion der bolschewistischen Revolution waren schon in den 1920er Jahren Motive für europäische Einigungspläne. Beide wurden durch die machtpolitischen Ergebnisse des Zweiten Weltkriegs verstärkt. Mit den USA als Weltführungsmacht und der Sowjetunion als stärkster Militärmacht des europäischen Kontinents verloren bisherige Interessendivergenzen zwischen europäischen Nationalstaaten an

4 Léon Blum: Blick auf die Menschheit, Zürich 1945, S. 104 (geschrieben 1941). Vgl. Wilfried Loth: Sozialismus und Internationalismus. Die französischen Sozialisten und die Nachkriegsordnung Europas 1940-1950, Stuttgart 1977

5 Blum: Blick auf die Menschheit, S. 108 - 109. 
Bedeutung - zugunsten des gemeinsamen Interesses an Autonomie und an der Vermeidung eines Konflikts zwischen den beiden Hauptsiegern des Krieges.

„Sich einigen oder untergehen“, wie es Clement Attlee 1939 wirksam formulierte, ${ }^{6}$ wurde damit zu einer in mehrfacher Hinsicht plausiblen Parole, schon unmittelbar nach dem Ersten Weltkrieg und in der Kritik an dem Ungenügen der Friedensordnung von Versailles, dann erneut, seit mit dem Münchner Abkommen deutlich wurde, dass die Ordnung von Versailles nicht mehr hielt, und verstärkt, seit sich 1943 der Sieg der Anti-Hitler-Koalition abzeichnete. Diese Parole faszinierte in den unterschiedlichsten politischen Lagern, sie verband über nationale Grenzen hinweg, und sie war - das muss gegen die spätere Fixierung auf den Ost-West-Konflikt und die daraus resultierende ahistorische Haltung gegenüber den 2004 beigetretenen Ländern betont werden - auch keineswegs nur ein westeuropäisches Phänomen. Die europäischen Verbände hatten ihre Sektionen genauso in Prag und in Budapest wie in Paris oder in Brüssel. ${ }^{7}$

Allerdings waren die Impulse, die sich aus den Defiziten der Nationalstaaten ergaben, nicht immer gleich stark, und sie wirkten auch nicht notwendigerweise immer in die gleiche Richtung. Die Frage der Friedenssicherung stellte sich 1918 noch anders als im Zeitalter der Weltmächte nach 1945; sie wurde in den unmittelbaren Nachkriegszeiten jeweils als drängender empfunden als nach Jahren der Gewöhnung an den Status quo. Die gemeinsame Notwendigkeit zur Einigung stand gegen real sehr unterschiedliche Befindlichkeiten und Bedürfnisse der $\mathrm{zu}$ einigenden Staaten, das übergreifende Interesse an wirtschaftlicher Einigung gegen sehr unterschiedliche wirtschaftliche Interessen im Detail. Will man den realen Gang der Integrationsgeschichte verstehen, muss man diese Inkongruenzen immer mit im Blick haben.

\section{Französische Initiativen}

Während des Zweiten Weltkriegs waren die meisten europäischen Exilregierungen mit Plänen zu regionalen Zusammenschlüssen in Europa beschäftigt, ebenso (wenn auch nur zeitweise) die britische Regierung und führende Vertreter des deutschen Exils und des Widerstands. Die Exilregierungen Polens und der Tschechoslowakei schlossen im Januar 1942 sogar ein förmliches Abkommen, in dem sie sich die Bildung einer ,Konföderation “ nach dem Krieg zusagten. Das Gleiche taten die griechische und die jugoslawische Exilregierung. Und auch Benelux, der wirtschaftliche Verbund der kleineren Weststaaten, beruht auf einer Vereinbarung, die während des Krieges getroffen wurde.

Die zahlreichen Einigungspläne, die in der Résistance überall in Europa entwickelt worden waren, verdichteten sich bei Kriegsende freilich nicht sogleich zu konkreter Einigungspolitik. Stalin blockierte jede Art von Zusammenschlüssen im östlichen Europa (so konsequent, dass die entsprechenden Pläne ganz aus der Erinnerung verschwunden sind). Gleichzeitig drohte jeder Schritt zur Einigung im Westen Europas die Spaltung des Kontinents in Ost und West zu vertiefen. Damit wurde es fragwürdig, mit Einigungsinitiativen dem Ziel der Friedenssicherung gerecht zu werden. Entsprechend schreckten jetzt viele vor substanziellen Entscheidungen zurück, auch und entscheidend die britische Regierung.

6 Rede vor der Labour-Fraktion am 08.11.1939, in: Clement R. Attlee: Labour's Peace Aims, London 1940, S. $12-13$.

7 Vgl. Walter Lipgens: Die Anfänge der europäischen Einigungspolitik 1945-1950, Band 1: 1943-1947, Stuttgart 1977; Walter Lipgens (Hrsg.): Documents on the History of European Integration, Band 1: Continental Plans for European Union 1939-1945, Berlin / New York 1985; Band 2: Plans for European Union in Great Britain and in Exile 1939-1945, Berlin / New York 1986; Michel Dumoulin (Hrsg.): Plans des temps de guerre pour l'Europe d'après-guerre 1940-1947, Brüssel 1995. 
Frankreich verstrickte sich zudem in die Forderung nach Abtrennung von Rhein und Ruhr vom deutschen Staatsverband, die bei den Nachbarn wenig Gegenliebe fand.

Für den politischen Durchbruch der Einigungsbewegung wurde darum die Zuspitzung des Ost-West-Konflikts zum Kalten Krieg entscheidend: Sie sorgte zum einen dafür, dass in der Frage der Spaltung des Kontinents eine klare Entscheidung getroffen wurde, an der die Europäer nicht mehr vorbeigehen konnten, und ließ zum anderen eine Einigung im Bereich des westlichen Europas noch dringlicher erscheinen - sei es, um die Gefahr einer Konfrontation zwischen USA und Sowjetblock abzuwehren, das westliche Europa gegen sowjetische Expansionsgelüste zu immunisieren oder vor einer amerikanischen Übermacht im Zeichen des Marshall-Plans zu schützen. Häufig wollte man das auch alles zugleich. Dann stellte sich mit der Einbeziehung des westlichen Deutschlands in das Eindämmungsprogramm auch das Problem der Schaffung eines Integrationsrahmens zur Kontrolle des deutschen Wiederaufstiegs in neuer Weise.

Sicherheit vor Deutschland konnte sich im Kalten Krieg weder auf eine gemeinsame Kontrolle durch die vier Siegermächte stützen noch auf eine einseitige Diskriminierung des neuen westdeutschen Staates. Die Deutschen konnten einen Preis für ihre neue Rolle als unverzichtbare Bündnispartner des Westens verlangen. Es war noch nicht einmal auszuschlieBen, dass sie sich, von den Fesseln des Besatzungsregimes erst einmal befreit, mit der Sowjetunion verbünden würden, die über den Schlüssel zur deutschen Einheit verfügte. Insbesondere diese Perspektive, die das Rapallo-Trauma der Westeuropäer wieder aufrührte, ließ die Schaffung supranationaler Strukturen im westlichen Europa jetzt, das heißt 1948, zu einem Dringlichkeitsprogramm werden. Frankreich sei davon überzeugt, hieß es in einer Note des Quai d'Orsay vom 4. Januar 1949, ,durch eine freiwillige Abtretung eines Teils staatlicher Souveränität und eine Assoziierung der Interessen dieser Staaten Deutschland binden und sich so die nötigen Sicherheitsgarantien verschaffen zu können. " ${ }^{8}$

Die französische Regierung hat die Einbindung der Deutschen seit dem vorherigen Sommer mit Nachdruck betrieben. Am 20. Juli 1948 - also knapp drei Wochen, nachdem die westdeutschen Ministerpräsidenten den Auftrag zur Vorbereitung der Gründung der Bundesrepublik erhielten - verlangte der französische Außenminister Georges Bidault in einem dramatischen Appell die Einberufung einer ,Europäischen Parlamentarischen Versammlung ' zum ,Meinungsaustausch' über die Probleme eines europäischen Zusammenschlusses und die Vorbereitung einer Wirtschafts- und Währungsunion. Diese Versammlung sollte, wie aus den französischen Regierungsakten hervorgeht, zunächst konsultativen Charakter haben, nach einer Verständigung über das Europa-Projekt aber bald eigene Entscheidungsvollmachten erhalten und damit ,den Kern einer föderativen Organisation Europas bilden."9

Weil die britischen Partner lange zögerten, ob sie sich diesem föderierten Europa anschließen sollten oder nicht, kam aus den Verhandlungen, die auf diese Initiative folgten, zunächst nicht mehr heraus als der Straßburger Europarat. In Frankreich und anderswo gab man sich damit freilich nicht zufrieden. Immer ungeduldiger drängte man auf den Ausbau

8 Zitiert nach Marie-Thérèse Bitsch: Le rôle de la France dans la naissance du Conseil de l'Europe, in: Raymond Poidevin (Hrsg.) : Histoire des débuts de la construction européenne (mars 1948 - mai 1950), Brüssel 1986, S. 165-198, hier S. 171. Zum Kontext der Diskussion der ersten Nachkriegsjahre vgl. Walter Lipgens / Wilfried Loth (Hrsg.): Documents on the History of European Integration, Band 3: The Struggle for European Union by Political Parties and Pressure Groups in Western European Countries 1945-1950, Berlin / New York 1988; Band 4: Transnational Organizations of Political Parties and Pressure Groups in the Struggle for European Union 1945-1950, Berlin / New York 1990.

9 Zitiert nach René Massigli: Une comédie des erreurs 1943-1956, Paris 1978, S. 157. 
des Europarats; und als sich herausstellte, dass die Briten dazu offensichtlich nicht zu bewegen waren, ergriff Bidaults Nachfolger Robert Schuman am 9. Mai 1950 die Initiative für einen Beginn des supranationalen Zusammenschlusses in Europa auch ohne britische Beteiligung. Das war der eigentliche politische Kern des Schuman-Plans - nicht die Idee einer europäischen Lenkungsbehörde für Kohle und Stahl und auch nicht die Hinwendung zu einer europäischen Lösung der deutschen Frage. Die Idee war schon seit den 1920er Jahren in verschiedenen Formen diskutiert worden, und die europäische Orientierung der französischen Deutschlandpolitik war seit mindestens zwei Jahren offenkundig. Indem Schuman unter dem Einfluss von Jean Monnet von Anfang an keinen Zweifel daran ließ, dass die französische Regierung auf supranationalen Kompetenzen der Montanbehörde bestehen würde, stellte er die Briten in unmissverständlicher Weise vor die Entscheidung, sich an den ersten Schritten des Einigungsprozesses zu beteiligen oder nicht. ${ }^{10}$

Die Entscheidung fiel buchstäblich in letzter Minute. Zwei Tage nach Schumans Ankündigung war eine Konferenz der Außenminister der drei Westalliierten angesetzt, auf der Amerikaner und Briten eine Aufhebung der Begrenzung der westdeutschen Stahlproduktion verlangen wollten. Eine Abschwächung des Besatzungsstatuts stand ebenfalls auf der Tagesordnung, und es war auch nicht mehr auszuschließen, dass über die Forderung des amerikanischen wie des britischen Generalstabs nach Aufstellung westdeutscher Truppen verhandelt werden musste. Sieben Wochen später, mit dem Beginn des Koreakrieges, wurde eben dies unvermeidlich.

Genau genommen war es damit für einen Erfolg des französischen Projekts eigentlich schon zu spät. Der Bundesrepublik boten sich jetzt, da man deutsche Truppen zur Verteidigung des Westens zu brauchen glaubte, nämlich Möglichkeiten zum Aufstieg auch ohne supranationale Organisation. Entsprechend verhärtete sich die deutsche Position in den Schuman-Plan-Verhandlungen, und Adenauers Politik dosierter Vorleistungen geriet unter zunehmenden innenpolitischen Beschuss. Um zu retten, was zu retten war, griff die französische Regierung zu einem Notbehelf, der das europäische Projekt für lange Zeit belasten sollte: zur Idee einer europäischen Armee. Nur so glaubte man die Deutschen noch davon abhalten zu können, abermals eine hegemoniale Rolle in Europa zu spielen, nur so schien auch der Schuman-Plan und damit die Idee einer Kontrolle der deutschen Schwerindustrie noch zu retten. „Die Umstände zwangen uns“, berichtete Jean Monnet später, ,die Zelte hinter uns abzubrechen. Die europäische Föderation wurde zu einem Nahziel." $" 11$

Das war logisch - eine europäische Armee setzte eine demokratisch legitimierten Politische Autorität voraus, die über den Einsatz dieser Armee entscheiden konnte. Dieses Projekt war aber gleichzeitig in psychologischer Hinsicht äußerst problematisch: Auf die nationale Souveränität ausgerechnet im militärischen Bereich zu verzichten, nur um gleichzeitig die Deutschen wieder mit Waffen auszustatten, dass war fünf Jahre nach dem Ende des natio-

10 Vgl. Raymond Poidevin: Robert Schuman, homme d'Etat 1886-1963, Paris 1986, S. 244-296; Klaus Schwabe (Hrsg.): Die Anfänge des Schuman-Plans 1950/51, Baden-Baden 1988; Ulrich Lappenküper: Der SchumanPlan. Mühsamer Durchbruch zur deutsch-französischen Verständigung, in: Vierteljahrshefte für Zeitgeschichte 3/1994, S. 403-445; Andreas Wilkens (Hrsg.): Le Plan Schuman dans l'histoire. Intérêts nationaux et projet européen, Brüssel 2004.

11 Jean Monnet: Erinnerungen eines Europäers, München/Wien 1978, S. 433. Zum Entscheidunsprozess, den der Koreakrieg auslöste, vgl. Norbert Wiggershaus: Die Entscheidung für einen westdeutschen Verteidigungsbeitrag 1950, in: Anfänge westdeutscher Sicherheitspolitik 1945-1956, Band 1: Von der Kapitulation bis zum Pleven-Plan, München/Wien 1982, S. 352-402; Wilfried Loth: Der Koreakrieg und die Staatswerdung der Bundesrepublik, in: Josef Foschepoth (Hrsg.): Kalter Krieg und Deutsche Frage, Göttingen 1985, S. 335-361; Rolf Steininger: Wiederbewaffnung. Die Entscheidung für einen westdeutschen Verteidigungsbeitrag. Adenauer und die Westmächte 1950, Erlangen 1989. 
nalsozialistischen Schreckens mehr, als man der Mehrheit der Westeuropäer zumuten konnte. Hinzu kam, dass ein großer Teil der Westeuropäer im Verteidigungsbereich gar keine europäische Autonomie wollte: Zum Schutz vor der Sowjetunion wie zum Schutz vor den Deutschen wollte man soviel amerikanische Präsenz in Europa wie nur irgend möglich: Eine europäische Einsatzzentrale für eine europäische Armee störte da nur.

Die ziemliche konfuse Diskussion, die sich aus diesen Empfindungen ergab, ${ }^{12}$ führte zu einem problematischen Ergebnis: dem Vertrag über die Schaffung einer Europäischen Verteidigungsgemeinschaft (der EVG), den Frankreich, die Bundesrepublik, die Benelux-Staaten und Italien am 27. Mai 1952 unterzeichneten. ,Europäisch“ war diese Gemeinschaft insofern nicht, als die Truppenführungsstäbe in operativer Hinsicht dem NATO-Hauptquartier unterstellt wurden; und eine supranationale ,Gemeinschaft " war es auch kaum, weil fast alle wesentlichen Entscheidungen, vom Erlass von Vorschriften bis zur Aufstellung des Budgets, von einstimmigen Voten des EVG-Ministerrats abhängig blieben. Der Versuch, dieses Kompromissgebilde dadurch auf solide Grundlagen zu stellen, dass man ihm eine Europäische Politische Gemeinschaft vorschaltete, scheiterte daran, dass die Niederländer etwas zur Vorbedingung machten, was die Franzosen partout nicht wollten: eine zusätzliche Wirtschaftliche Gemeinschaft.

Der EVG-Vertrag stieß dann auf heftige Kritik, vor allem in Frankreich. Nach vielen Monaten leidenschaftlicher Auseinandersetzungen und vergeblicher Rettungsversuche wurde er Ende August 1954 von der französischen Nationalversammlung zu Fall gebraucht. Für viele Anhänger des europäischen Einigungsgedankens war dies ein harter Schlag. Viele sind auch heute noch davon überzeugt, dass sich das Einigungsprojekt nie mehr davon erholt hat. Schaut man jedoch etwas genauer hin, wird deutlich, dass die Gegner der EVG keineswegs nur rückständige Nationalisten waren, und dass ihr Scheitern auch nicht die Rückkehr zu einer nationalstaatlichen Normalität nach der europäischen Aufbruchstimmung der Nachkriegszeit signalisierte. Genaue Untersuchungen des Meinungsspektrums und des Abstimmungsverhaltens zeigen, dass die Idee eines europäischen Zusammenschlusses in den Ländern der Sechs grundsätzlich mehrheitsfähig blieb, auch wenn über die Art ihrer Ausführung die Meinungen auseinander gingen. ${ }^{13}$

\section{Der Kompromiss der Römischen Verträge}

Nach dem Scheitern der französischen Flucht nach vorn musste nun allerdings ein Ausweg aus der Krise der Gemeinschaftsbildung gefunden werden, der auch in der Konkretisierung mehrheitsfähig war. Auf sich allein gestellt war die Europäische Gemeinschaft für Kohle und Stahl auf Dauer nicht überlebensfähig, und sie genügte auch nicht - weder zur dauerhaften Einbindung der Deutschen, noch zur Behauptung europäischer Autonomie gegenüber der amerikanischen Führungsmacht und schon gar nicht zur Befriedigung der verschiedenen wirtschaftlichen Bedürfnisse. Die Suche nach einem solchen Ausweg führte erneut zu einem komplizierten Kompromiss - diesmal freilich zu einem, der sich langfristig als tragfähig erweisen sollte.

12 Vgl. dazu insbesondere Klaus A. Maier: Die internationalen Auseinandersetzungen um die Westintegration der Bundesrepublik Deutschland und um ihre Bewaffnung im Rahmen der Europäischen Verteidigungsgemeinschaft, in: Anfänge westdeutscher Sicherheitspolitik 1945-1956, Band 2: Die EVG-Phase, München / Wien 1990, S. 1-234.

13 Siehe Wilfried Loth: Die EVG und das Projekt der Europäischen Politischen Gemeinschaft, in: Rainer Hudemann / Hartmut Kaelble / Klaus Schwabe (Hrsg.): Europa im Blick der Historiker, München 1995, S. 191-201; sowie die Beiträge in: Gilbert Trausch (Hrsg.): Die europäische Integration vom Schuman-Plan bis zu den Verträgen von Rom, Baden-Baden 1993. 
Man kennt diesen Kompromiss als die Kombination von EURATOM und EWG in den Römischen Verträgen vom 25. März 1957. Er beruhte darauf, dass Frankreich jetzt die von den Niederländern geforderte Wirtschaftsgemeinschaft akzeptierte - freilich nur für eine fernere Zukunft, die über mehrere Etappen erreicht werden sollte - während die europäischen Partner die Atomgemeinschaft hinnahmen, für die sich außer den französischen Technokraten niemand erwärmen konnte. Wichtig für sein Zustandekommen war, dass Spitzenbeamte und Politiker, die aus der Erfahrung mit dem Desaster der EVG ihre Lehren gezogen hatten, die Interessendivergenzen nach Möglichkeit vor dem allgemeinen Publikum verbargen und in den komplizierten Vertragswerken vieles offen ließen. Insbesondere drei Politiker sind hier zu nennen: der belgische Außenminister Paul-Henri Spaak, der als Vorsitzender einer von den Außenministern der Sechs eingerichteten Kommission den Konsens der Experten gegen politische Einflüsse abschirmte; der französische Ministerpräsident Guy Mollet, der sich Schritt für Schritt eine Mehrheit für das Projekt in der französischen Nationalversammlung erarbeitete; und Bundeskanzler Konrad Adenauer, der die deutsche Beteiligung gegen ökonomische Interessen und ordnungspolitische Bedenken durchsetzte. ${ }^{14}$

Für Wirtschaftsminister Ludwig Erhard war ein Gemeinsamer Markt der Sechs mit Außenzoll und Steuerungskompetenzen ,volkswirtschaftlicher Unsinn“. Die deutsche Industrie- und Finanzwelt stand ihm ziemlich reserviert gegenüber, gingen doch gerade einmal 25 Prozent des Exports der Bundesrepublik in die anderen EWG-Länder. ${ }^{15}$ Für Adenauer aber hatte die Politik einmal mehr Vorrang vor der Wirtschaft: Für ihn ging es darum, die Bindung der Bundesrepublik an den Westen abzusichern, die ihm mit der NATO-Mitgliedschaft allein noch längst nicht auf Dauer garantiert schien. Zudem schwebte ihm auch eine Sicherheitsgemeinschaft Westeuropas vor - für den Fall, dass sich die USA von der Verteidigung Westeuropas abwenden sollten, ein Alptraum, der ihn nie verließ. Darin traf er sich mit den Vorstellungen Mollets, der die nun schon traditionelle Sorge um die Einbindung der Deutschen mit der Hoffnung auf eine größere Unabhängigkeit der Europäer in der Weltpolitik verband. ${ }^{16}$

Sieht man einmal von dem niederländischen Interesse an einer Zollunion und einem europäischen Agrarmarkt für den Absatz niederländischer Produkte ab, so war die Europäische Wirtschaftsgemeinschaft also vorwiegend ein politisches Unternehmen: In wirtschaftlicher Hinsicht sowohl für Frankreich als auch für die Bundesrepublik (also für die beiden Hauptbeteiligten) problematisch, aber von beiden um der politischen Zielsetzung willen akzeptiert. Einbindung der Deutschen und Autonomie gegenüber den USA waren ihre eigentlichen Ziele - freilich auch ihre heimlichen; und darin lag das Problem der 1957 gefundenen Konstruktion. Weil die wirtschaftlichen Interessen nach wie vor divergierten, konnte sich die Gemeinschaft nur dann einigermaßen harmonisch entwickeln, wenn man sich der zugrundeliegenden politischen Notwendigkeiten bewusst war. Davon aber war in den Vertragstexten nicht die Rede; und Adenauer ebenso wie Mollets Nachfolger hüteten sich mit Rücksicht auf die Befindlichkeit der öffentlichen Meinungen, allzu deutlich darauf hinzuweisen.

14 Vgl. Hanns Jürgen Küsters: Die Gründung der Europäischen Wirtschaftsgemeinschaft, Baden-Baden 1982; Peter Weilemann: Die Anfänge der Europäischen Atomgemeinschaft. Zur Gründungsgeschichte von Euratom 1955-1957, Baden-Baden 1983; Enrico Serra (Hrsg.): Il rilancio dell'Europa e i Trattati di Roma, Mailand 1989; Wilfried Loth: Deutsche und französische Interessen auf dem Weg zu EWG und Euratom, in: Andreas Wilkens (Hrsg.): Die deutsch-französischen Wirtschaftsbeziehungen 1945-1960, Sigmaringen 1997, S. 178187; Wilfried Loth: Vor 40 Jahren: Die Verhandlungen über die Römischen Verträge, in: integration 1/1997, S. 1-12.

15 Siehe Wilfried Loth: Deutsche Europa-Konzeptionen in der Gründungsphase der EWG, in: Serra: Rilancio, S. 585-602.

16 Vgl. François Lafon: Guy Mollet. Itinéraire d'un socialiste controversé (1905-1975), Paris 2006, S. 491-499. 
In der Praxis erwies sich die EWG aufgrund des gestuften Integrationsprogramms, das im Detail immer noch zu verhandeln war, zunächst als ein vorzügliches Instrument sozialverträglicher Modernisierungsförderung. Frankreich konnte die Wettbewerbsfähigkeit mit der Bundesrepublik dank der Integrationssteuerung deutlich schneller erreichen als zur Zeit der Vertragsverhandlungen erwartet; Belgien sah sich bei der Bewältigung der Krise seiner Montanindustrie unterstützt, Italien im Umbruch zur Industriegesellschaft gefördert. Auf diese Weise gewannen die ökonomischen Motive für den Zusammenschluss nachträglich an Gewicht. Der Export in die anderen Mitgliedsländer stieg im ersten Jahrzehnt der Gemeinschaft auf das Dreieinhalb- bis Vierfache, im Falle Italiens sogar auf das Sechseinhalbfache. 1969, im Jahr nach der vorzeitigen Vollendung der Zollunion, die durch die raschen Modernisierungserfolge möglich wurde, erreichte er das Viereinhalb- bis Fünfeinhalbfache, in Italien das Achtfache. ${ }^{17}$ Mit dem Beitritt Großbritanniens, Irlands und Dänemarks zum 1. Januar 1973, der aus wirtschaftlichen Gründen unumgänglich wurde, ${ }^{18}$ wurde die ökonomische Motivation weiter gestärkt.

Die politischen Motive für den Zusammenschluss gingen dadurch nicht verloren. Es bedurfte aber weiterhin der Verständigung über diese impliziten Zielsetzungen, und diese gelang bekanntlich nicht immer. Statt sich in akademischen Diskussionen über die ,Finalität" der Gemeinschaft zu verzetteln, wären die Verantwortlichen der heutigen Europäischen Union gut beraten, den Dialog über die politischen Zielsetzungen zu forcieren - sowohl zwischen den Regierungen als auch zwischen den europäischen Eliten und den Bürgern der Union. ${ }^{19}$

17 Berechnungen bei N. Piers Ludlow: The European Community and the Crises of the 1960s. Negotiating the Gaullist challenge, London / New York 2006, S. 53.

18 Ludlow: European Community, S. 146-198; vgl. auch Oliver J. Daddow (Hrsg.): Harold Wilson and European Integration. Britain's Second Application to Join the EEC, London / Portland, Oregon 2003.

$19 \mathrm{Zu}$ den institutionellen Defiziten des Vertragswerks von Rom, die einen solchen Dialog bislang behindert haben, siehe Guido Thiemeyer: Die Ursachen des „Demokratiedefizits“ der Europäischen Union aus geschichtswissenschaftlicher Perspektive, in: Wilfried Loth (Hrsg.): Das europäische Projekt zu Beginn des 21. Jahrhunderts, Opladen 2001, S. 27-47; Wilfried Loth: Der Post-Nizza-Prozess und die Römischen Verträge, in: integration 1/2002, S. 45-52. 